core-set of 17 arteries and respectively represent the sum of stenosis and dilation scores in individual arteries ${ }^{1}$

Objectives: To use ASS, ADS and the stenosis and dilation scores of individual arteries to describe a cohort of LVV patients and identify heterogeneity between patients.

Methods: The ASS, ADS and individual artery scores have been derived from 110 LVV (81 TA, 29 GCA) patients. Model-based clustering optimising the Bayesian Information Criterion and principal component analysis were performed.

Results: Arterial involvement was shown to be differed in GCA and TA: TA has higher ASS than GCA (median, IQR: 20, 11-29 Vs 5, 0-11; $p<0.001$ ) and lower ADS $(0,0-5$ Vs $6,0-13 ; p=0.019)$. The scatterplot of ASS and ADS revealed incomplete overlap of arterial involvement in GCA and TA. No differences were seen in TA with disease onset before or after $40 \mathrm{yrs}$. Age at onset, ASS and ADS did not correlate in TA, suggesting stenotic and aneurysmal arterial remodelling are independent. In GCA, ASS and ADS were negatively correlated $(\rho=-0.401 ; p=0.031)$ and ADS correlated with age at onset $(\rho=0.383 ; p=0.040)$, suggesting the existence of a biologic "switch" between arterial stenosis and dilation, regulated by age at onset.

We accounted for geographical distribution of lesions by evaluating the scores of individual arteries with correspondence analysis. Arterial involvement was symmetric with tripolar segregation: stenosis in the supra-aortic branches, stenosis in the aorto-abdominal district and arterial dilation (Fig $1 \mathrm{~A})$. When patients exhibited the first two components, three different clusters were recognised (Fig 1B), with different ASS, ADS and damage as assessed by the TA damage score $(p<0.001$ for all tests). 27/29 (93\%) of GCA patients were included in cluster 1 and 3 , while $77 / 81$ (95\%) of TA clustered in 2 and 3 . Of interest, density graphs showed (i) a different distribution of arterial involvement in GCA and TA (Fig $1 C-D$ ), and (ii) potential for identification of novel disease subsets (2 in GCA and 3 in TA). A comparable distribution was seen in TA with onset before or after 40 yrs (Fig 1E). Lastly, when patients with disease onset after 50 yrs (11 TA, 28 GCA) were studied, a trimodal distribution was observed, suggesting discrete phenotypes of arterial involvement exist, rather than a continuum (Fig $1 \mathrm{~F}$ ).
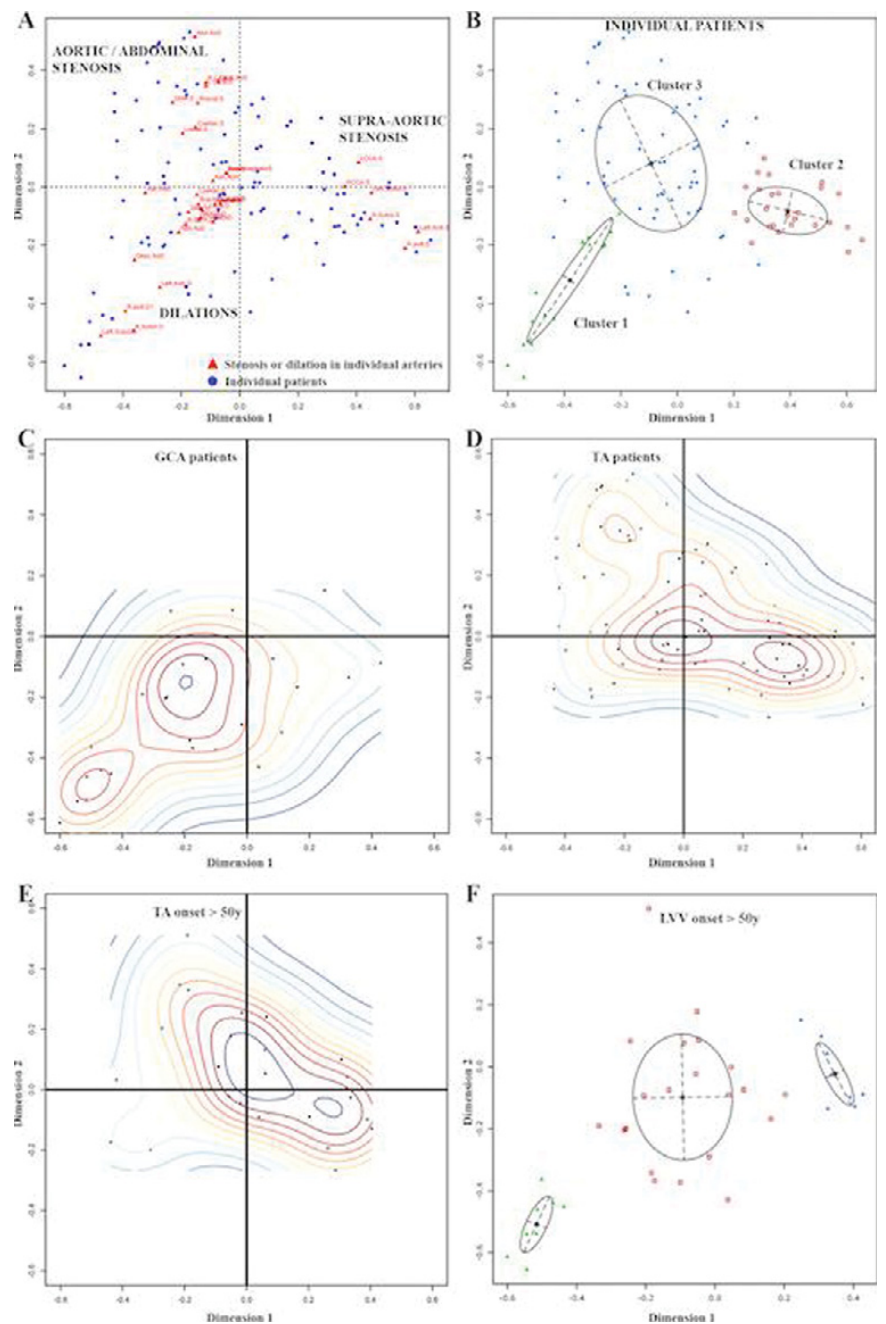

Conclusions: Arterial involvement differs in TA and GCA, although some overlap exists. Elderly TA is similar to juvenile TA, while a potential biologic "switch", yet to be identified, regulating the final outcome of arterial remodelling and influenced by ageing, is present in GCA. Three main patterns of arterial involvement appear to exist. LVVs represent the composition of different discrete subsets rather than a phenotypic continuum.

References:

[1] : Tombetti et al. EULAR 2015 Poster FRI0258.

Disclosure of Interest: None declared

DOI: 10.1136/annrheumdis-2017-eular.6386

\section{THU0300 CENTRAL NERVOUS SYSTEM INVOLVEMENT IN GRANULOMATOSIS WITH POLYANGIITIS (WEGENER) IN A LARGE SERIES OF PATIENTS WITH ANCA-ASSOCIATED VASCULITIDES (AAV).REVAS STUDY-GEAS-SEMI}

R. Solans-Laqué ${ }^{1}$, M. Rodriguez-Carballeira ${ }^{2}$, M. Castillo ${ }^{3}$, G. Fraile $^{4}$, J. Rios $^{5}$ X. Solanich ${ }^{6}$, F. Martinez-Valle ${ }^{1}$, L. Saez ${ }^{7}$, F. Pasquau ${ }^{8}$, E. Fonseca ${ }^{9}$ on behalf of REVAS Study group. ${ }^{1}$ Internal Medicine, Vall Hebron University Hospital, Barcelona; ${ }^{2}$ Internal Medicine, Mutua Hospital, Terrassa; ${ }^{3}$ Internal Medicine Virgen Rocio Hospital, Sevilla; ${ }^{4}$ Internal Medicine, Ramon y Cajal Hospital; ${ }^{5}$ Internal Medicine, la Paz University Hospital, Madrid; ${ }^{6}$ Internal Medicine, Bellvitge University Hospital, Hospitalet; ${ }^{7}$ Internal Medicine, Miguel Servet University Hospital, Zaragoza; ${ }^{8}$ Internal Medicine, Marina Baixa Hospital, Alicante; ${ }^{9}$ Internal Medicine, Cabueñes Hospital, Gijon, Spain

Background: GPA is a necrotizing systemic vasculitis that usually involves ENT, lung and kidneys. Neurological manifestations appear in $25-50 \%$ of patients, usually involving peripheral nervous system. CNS involvement, has been reported in only $7-11 \%$ of cases

Objectives: to describe the clinical features and outcome of patients with GPA and CNS involvement in a large series of patients with AAV

Methods: multicenter retrospective-longitudinal study that encompassed patients diagnosed with AAV between Jan 1995 and Nov 2014 in 21 Centres from Spain (REVAS Study). Statistical anlysis was performed using SSPS vs20 package Results: 455 patients (188 GPA, 167 MPA and 100 EGPA) were included. Mean age at diagnosis was $55.7 \pm 17.2 y$. ANCA were positive in $86.8 \%$ of cases (35.8\% C-ANCA, 51\% P-ANCA). Median time to diagnosis was 4 weeks (IQR 10). Median follow-up time was 80 months (IQR 105). Neurological involvement was documented in 156 (34.5\%) patients, but only $33(7.3 \%)$ presented CNS involvement at disease onset. From those patients, $20(60.6 \%)$ had GPA. Mean age at diagnosis of patients with GPA and CNS involvement was $51.1 \pm 16.7 \mathrm{y}$. ANCA were positive in all cases (15 C-ANCA-PR3, 5 P-ANCA-MPO). Headache was the main neurological symptom $(60 \%)$ followed by sensory $(45 \%)$ and motor impairment $(35 \%)$. MRI and/or angio-CT scan were performed in all cases. Cerebral ischaemic lesions were observed in 10 patients, and granulomatous lesions in 9 , including pachymeningitis $(n=6)$, spinal cord pachymeningitis $(n=2)$ and isolated granulomatous lesions $(n=1)$. Lumbar puncture was performed in 8 $(40 \%)$ patients and revealed CSF abnormalities in 70 . Diagnosis was confirmed by meningeal biopsy $(n=2)$, ENT biopsies $(n=5)$ and renal biopsy $(n=2)$ in patients with CNS granulomatous lesions, and by renal, pulmonary or peripheral nerve biopsy in patients with CNS ischemic lesions. Headache was predominant in patients with granulomatous lesions, while sensory and motor impairment were predominant in patients with ischemic lesions. Mean BVAS at disease onset was $29.2 \pm 9.7$, significantly higher than in GPA total cohort (18.2 \pm 9.2$)$. Renal involvement was more common in patients with ischaemic lesions than in those with granulomatous ( $80 \%$ vs. $40 \%, \mathrm{p}<0.001)$, and ENT involvement in patients with granulomatous forms $(70 \%$ vs. $50 \%, p<0.005)$. Most patients $(70 \%)$ received oral CF for induction therapy. Two patients received rituximab. For maintenance therapy, $25 \%$ of patients received AZA, $20 \%$ MMF and the remaining CF. $70 \%$ of patients received TM-SX. During follow-up, $58.8 \%$ and $40 \%$ of patients developed bacterial and opportunistic infections, respectively. Infections were related to oral CF therapy $(p=0.029)$. Long-term neurological sequelae were noted in patients with ischaemic lesions (40\%) and spinal cord pachymeningitis (100\%)

Conclusions: Patients with GPA and CNS involvement have more severe disease at presentation and more treatment-related side effects than patients without CNS involvement. Long-term neurological sequelae are more frequent in patients with ischaemic lesions and spinal cord pachymeningitis

Disclosure of Interest: None declared

DOI: 10.1136/annrheumdis-2017-eular.2520 